\title{
Outcomes of Children with Severe Acute Malnutrition in a Tribal Day- care Setting
}

\author{
Vandana Prasad, Dipa Sinha, Priyanka Chatterjee and Rajkumar Gope \\ From Action Against Malnutrition (AAM) Consortium, New Delhi, India.
}

Correspondence to:

Dr Vandana Prasad, 2,42, First Floor, Sarvapriya Vihar, New Delhi 110067 , India.chaukhat@yahoo.com Received: December 12, 2016; Initial review: March 14, 2017; Accepted: November 27, 2017.
Objective: To describe the nutritional outcomes of children with severe acute malnutrition (SAM) in a village-level intervention. Methods: This observational longitudinal study on 179 children aged $<3$ years was conducted in seven tribal blocks of Central and Eastern India with SAM managed in a comprehensive day care program. Results: $76 \%$ children with SAM showed improvement over a 4-6 months period, with $37 \%$ shifting to normal anthrometric status. There was a significant shift in Z scores. Conclusion: This community-based intervention showed fair results for management of children with SAM at village level.

Keywords: Anthropometry, Nutritional status, Protein energy malnutrition, Treatment.

Published online: December 14, 2017. PII:S097475591600100

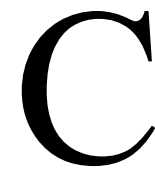

ommunity-based management of malnutrition remains a challenge for India with limited numbers of Nutritional Rehabilitation Centers (NRC) providing facility-based care for a small proportion of children with Severe Acute Malnutrition (SAM) [1,2]. Action Against Malnutrition (AAM) is a model that offers comprehensive care to children below the age of 3 years through day-care facilities, and works closely with women on nutrition-action through Participatory learning and action (PLA) cycles, home visits, and close liaison with related government facilities and services. The program thus refers to the UNICEF framework for nutrition that states the proximal determinants of nutrition as 'care', 'health' and 'food' [3].

While some program outcomes based on similar data have been published previously [1], limitations included small sample size and possible reference of seasonality. This study seeks to circumvent these limitations by nutritional outcomes of a larger set of children across seasons, considering the same intervention over a similar period of time (4 to 6 months).

\section{METHODS}

AAM is being collaboratively implemented by Public Health Resource Society (PHRS), Ekjut, Child In Need Institute, Chaupal, and IDEA, in seven blocks spread across the Indian states of Bihar, Chhattisgarh, Jharkhand and Odisha. The selection of areas was further based on vulnerability-mapping, and analysis through a formal baseline survey in the program areas confirmed high prevalence of wasting and stunting. An ethical approval for the entire program, including anthropometry, data analysis and research, was obtained from the Institutional Ethics Committees of PHRS and Ekjut. The main interventions of the program were:

1. Care: Daycare by trained local women. Comprehensive Early Childhood Care Development, child safety and protection.

2. Nutrition: (a) Supervised feeding using local foods with inputs for protein-sufficiency and caloriedensity (Eggs, Adapted SAT mix / Nutrimix / Sattu; $70 \%$ caloric requirement and $15 \mathrm{~g}$ of protein per day); (b) Building capacities of family and community for behavior change through PLA; and (c) growth monitoring, early community-level action triggered by growth faltering.

3. Health: promotion and prevention, focus on handwashing, hygiene, safe water, oral rehydration salt (ORS) solution for treatment of diarrhea, smoke-free environment, referral to NRC (as per government protocols) and PHC.

In the AAM crèches, every child's weight was recorded monthly and height once in six months besides at the time of admission. In April 2013, it was decided that for the purpose of uniformity across crèches, heights would be measured at entry point and in the months of May and November each year for all children, irrespective of their date of admission. The outcomes of 45 SAM children were reported early in the program as part of a cohort of 587 children in all categories [1]. These 45 children are also included in the current analysis. 
For this analysis, data for children (age $<3$ years) for whom valid anthropometric measurements (both height and weight) were available for at least two points in time have been considered. Such data were available for 2768 children. Of these children, we selected those children for whom anthropometric measurements were available with a gap of four to six months. Of 1615 such children, $179(11 \%)$ children had SAM (weight-for-height $Z$ score $(\mathrm{WHZ})<-3$ ) according to their first weight for height measurement.

The data were recorded using a customized software for the program and, the data analysis was carried out using Stata (version 13). $Z$-scores were calculated using the growth standards provided by WHO [4]. The usual WHO cut-offs for implausibility were applied.

\section{RESULTS}

Out of the 179 included children ( 100 males), $86 \%$ belonged to scheduled tribe and $5 \%$ to scheduled caste categories. Most fathers (81\%) and mothers (57\%) worked as casual agricultural or non-agricultural labourers. Two-thirds held Below Poverty Line /Antodyaya cards and 93\% lived in kutchha houses; $70 \%$ of the mothers had no schooling at all.

The average age of the children at the time of first measurement was 14.5 months. For 129 children, the first measurement coincided with their time of admission to the crèche. For the rest, average duration of stay of the children before the time of their first available and valid weight and height measurement was 1.9 months. For 63 children, the gap between the two measurements was 4 months, for 48 children the gap was 5 months, and for 68 children the gap was 6 months. All the children were referred to nutritional rehabilitation centers through the Anganwadi worker / ASHA as per government protocols. Only 30 of them were ever admitted there.

Table I presents the nutritional status of the 179 SAM children after a period of 4 to 6 months. During this period, about three-fourths of the children improved their status from SAM to normal or moderate malnutritional. The mean $Z$-score for these children increased significantly

TABLE I Change IN Weight-For-Height Status of Children With Severe Acute Malnutrition

\begin{tabular}{lccr}
\hline $\begin{array}{l}\text { Weight- } \\
\text { for- } \\
\text { height status }\end{array}$ & $\begin{array}{c}4 \text { to } \\
6 \mathrm{mo} \\
(n=179)\end{array}$ & $\begin{array}{c}* \text { Any } \\
\text { duration } \\
(n=179)\end{array}$ & $\begin{array}{r}\text { \# Over at } \\
\text { least 6 mo } \\
(n=114)\end{array}$ \\
\hline Normal & $66(37 \%)$ & $99(55 \%)$ & $73(64 \%)$ \\
Moderately wasted & $70(39 \%)$ & $57(32 \%)$ & $33(29 \%)$ \\
Severely wasted & $43(24 \%)$ & $23(13 \%)$ & $8(7 \%)$ \\
\hline
\end{tabular}

Values in no. (\%); *mean 13 mo; " mean 17 mo.
$(P<0.001)$ from $-3.63(0.51)$ to -2.3 (1.07). Table I also presents the change in wasting status based on the first and last anthropometric measurements of these children in the program. Only 23 (13\%) children continued to remain in the category of SAM at the end of the analysis period. Of these 23 children, the duration between the two measurements for 15 children was between 4 to 6 months. 65 children graduated from the programme or were unavailable for measurement after 4-6 months. The outcomes of these children are not statistically different from the overall 179 .

The improvement in $Z$-scores was statistically significant $(P<0.001)$ in both seasons in which the first measurement of children was taken. The change in the $Z$-scores for these two groups did not show any significant difference between the two seasons (Table II).

\section{Discussion}

This village-level based study documented that almost three-fourth of the children with SAM show improvement in their WHZ category over a period of 4-6 months, when managed in a comprehensive community-based program.

Most other observational studies on community-based management of SAM in India have reported the nutritional outcomes of a smaller number of children [5-7]. Primarily facility-based interventions with outreach facilities to community, such as the Darbhanga model [2] have been able to cover far larger numbers with distinctly better nutritional outcomes. However, the Darbhanga model was challenged by very high dropout rates, no intervention for prevention or early detection, and questionable sustainability. In the recently published results of a rigorous randomized control trial [8] comparing the efficacy of three different feeding regimens for SAM, the recovery rate in the most efficacious arm was also only $57 \%$, further dwindling to $17 \%$ ( $29 \%$ remaining SAM) 16 weeks after the end of the treatment [8]. In our study, gains continued with time with proportion of children with SAM dropping with increasing duration of intervention.

TABLE II WEIGHT-FOR-HEIGHT STATUS IN CHILDREN WITH SAM

\begin{tabular}{llr}
\hline Weight-for-Height & \multicolumn{2}{c}{ Season } \\
\cline { 2 - 3 } Status & $\begin{array}{l}\text { May to November } \\
(n=91)\end{array}$ & $\begin{array}{r}\text { November to May } \\
(n=88)\end{array}$ \\
\hline Normal & $40(44 \%)$ & $26(29.6 \%)$ \\
Wasted & $32(35 \%)$ & $38(43.2 \%)$ \\
Severely wasted & $19(21 \%)$ & $24(27.3 \%)$ \\
Initial WHZ & $-3.65(0.52)$ & $-3.61(0.49)$ \\
Final WHZ & $-2.21(1.08)$ & $-2.38(1.02)$ \\
\hline WHZ: Weight-for-height Z $Z$ & score; Values in No. (\%); ${ }^{*} P$ values \\
$<0.001$ in both seasons &
\end{tabular}




\section{What This Study Adds?}

- There is improvement in nutritional outcomes of children with SAM through a village-based comprehensive daycare program in a tribal setting.

The limitation of this study is that these results are based on program data from the crèche component of the comprehensive program; the outcomes represent the net and 'best case' outcomes of all three strategies of AAM and not just the crèche program. The project was located in medically underserved areas and the referrals to the government health system were not sufficient to meet the many immediate medical needs of the children in crèches. The program itself was not able to offer direct medical services or facility-based care and this was a limiting factor in nutritional outcomes and overall well-being of attending children. Every crèche could not be provided with stadiometers/infantometers. The quality of data collected by supervisors was monitored through a protocol of quality checks following rigorous capacitybuilding. However, as with most field and facility programs on malnutrition, we did not formally analyze inter- and intra-observer reliability [1].

This study describes the observations of the AAM program, across seasonal influences, on nutritional outcomes in a community setting, and confirms the findings of other studies. The findings of this study reinforce the need for daycare programs for children that can accommodate all the requirements of a comprehensive community-based program for malnutrition.

Acknowledgements: This study is based on the collaborative project "Community-based Action on Malnutrition among Children Under Three Years: A multi-strategy intervention" run by the following organizations: CINI, Chaupal, Ekjut, IDEA and Public Health Resource Society. We are grateful for the leadership of Dr. Prasanta Tripathy, Dr. Ganapathy Murugan, Dr. Suranjeen Prasad Pallipamula, Ms. Jayeeta Chowdhury, Ranjan K Panda, Sulakshana Nandi, Dr. Audrey Prost, Haldhar Mahto, Gangaram Paikra and Digvijay Kumar. We would also like to thank Biswanath Dasgupta for his insightful comments on the paper. We thank all collaborating organizations for agreeing to use the
Project MIS data for this study.

Contributors: VP: conceptualized and led the study. All authors participated in data analysis, manuscript writing and approved the final draft.

Funding: Tata Social Welfare Trust; Competing interests: None stated.

\section{REFERENCES}

1. Prasad V, Sinha D. Potentials, experiences, and outcomes of a comprehensive community based program to address malnutrition in tribal India. Int $\mathrm{J}$ Child Health Nutr. 2015;4:151-62.

2. Burza S, Mahajan R, Marino E, Sunyoto T, Shandilya C, Tabrez M, et al. Community-based management of severe acute malnutrition in India: New evidence from Bihar. Am J Clin Nutr. 2015;101:847-59.

3. Strategy for Improved Nutrition of Women and Children in Developing Countries, United Nations Children's Fund 1990.

4. WHO Child Growth Standards: Length/height-for-age, Weight-for-age, Weight-for-length, Weight-for-height and Body Mass Index-for-age: Methods and Development. WHO Multicentre Growth Reference Study Group, WHO 2006.

5. Ashworth A. Efficacy and effectiveness of communitybased treatment of severe malnutrition. Food Nutr Bull. 2006;27:S24-48.

6. Shewade H, Patro B, Bharti B, Soundappan K, Kaur A, Taneja N. Effectiveness of indigenous ready-to-use therapeutic food in community-based management of uncomplicated severe acute malnutrition: A randomized controlled trial from India. J Trop Pediatr. 2013;59:393-8.

7. Patel D, Gupta P, Shah D, Sethi K. Home-based rehabilitation of severely malnourished children in resource poor setting. Indian Pediatr. 2010;47:694-701.

8. Bhandari N, Mohan SB, Bose A, Iyengar SD, Taneja S, Mazumder S, et al. Efficacy of three feeding regimens for home-based management of children with uncomplicated severe acute malnutrition: a randomised trial in India. BMJ Global Health. 2016;1:e000144. 